Titles and abstracts of identified records were assessed independently by two reviewers. Full texts of all potentially relevant papers were retrieved. Two reviewers assessed each full text and reasons for exclusion were recorded. Differences in opinions were resolved by a third reviewer.

Methodological quality was assessed by two reviewers independently as low, medium or high in terms of trustworthiness and reliability of findings. This was done using criteria developed by the EPPI-Centre. Full texts will be opened in NVivo and analysed using thematic synthesis, as described by Thomas and Harden (2008). The certainty of review findings will be assessed using the Confidence in the Evidence from Reviews of Qualitative Research (CERQual) approach.

Results 19230 records were identified for title and abstract screening. 284 full-texts were screened, with 30 included in the final sample.

Conclusion This review is due for completion in June 2017. Findings will inform development of an adolescent weight management intervention in Coventry, West Midlands.

To date, no systematic review has focused on adolescent views of weight management interventions. The views of adolescents should be taken as a starting point when planning future initiatives.

\section{P94 ASSOCIATIONS BETWEEN MATERNAL PREGNANCY, SOCIAL AND LIFESTYLE CHARACTERISTICS AND OFFSPRING BLOOD PRESSURE AT AGE 4/5 IN WHITE BRITISH AND PAKISTANI ORIGIN PARTICIPANTS IN THE BORN IN BRADFORD STUDY}

1J West ${ }^{*},{ }^{1} \mathrm{G}$ Santorelli, ${ }^{1} \mathrm{P}$ Collings, ${ }^{1} \mathrm{~J}$ Wright, ${ }^{2,3} \mathrm{D}$ Lawlor. ${ }^{1}$ Born in Bradford, Bradford Institute for Health Research, Bradford, UK; ${ }^{2}$ School of Social and Community Medicine, University of Bristol, Bristol, UK; ${ }^{3}$ MRC Integrative Epidemiology Unit, University of Bristol, Bristol, UK

\subsection{6/jech-2017-SSMAbstracts. 195}

Background South Asians have an increased risk of stroke and coronary heart disease (CHD) compared to white European populations. High blood pressure (BP) is an important risk factor for CHD and higher diastolic BP has previously been reported in both South Asian adults and children. Our aim was to examine whether maternal pregnancy, social and lifestyle characteristics are associated with BP at age $4 / 5$ and contribute to these differences.

Methods Born in Bradford is a prospective study of children born to 12453 mothers between 2007 and 2010 in Bradford, UK. All mothers completed an oral glucose tolerance test in pregnancy and provided detailed social and lifestyle data. We present data from 2129 White British and 2528 Pakistani mother/offspring pairs from the Born in Bradford study. Associations of BMI, gestational diabetes (GD), fasting and post load glucose, maternal hypertension (HDP), smoking in pregnancy and maternal education with offspring systolic and diastolic BP at age $4 / 5$ were examined. We adjusted our results for sex and age at BP measurement.

Results Pakistani children had lower systolic (mean difference $-0.1695 \% \mathrm{CI}-0.79,0.47$ ) but higher diastolic (mean difference $1.3895 \%$ CI 0.74, 2.03) compared to White British children. In Pakistani children maternal BMI and HDP were strongly associated with higher systolic and diastolic BP but the effect was minimal and weak in White British children. Associations of maternal glucose and BP were consistent with the null hypothesis in both groups. Smoking and education were not associated with BP in either group.

Conclusion Ethnic differences in systolic and diastolic BP are present in children at age $4 / 5$. Consistent with findings in adults, Pakistani origin children have on average lower systolic and higher diastolic BP compared to White British children. Maternal BMI and HDP influence BP in Pakistani children but have minimal effect in White British children. These results suggest a role for some maternal pregnancy and lifestyle exposures in ethnic differences in systolic and diastolic $\mathrm{BP}$ at age $4 / 5$.

\section{P95 ETHNIC VARIATIONS IN CLUSTERING OF ADOLESCENT HEALTH RISK BEHAVIOURS: LATENT CLASS ANALYSIS}

${ }^{1} \mathrm{~A}$ Cassidy*, 'O Molaodi, ${ }^{1} \mathrm{M}$ Green, ${ }^{1} \mathrm{~L}$ Moore, ${ }^{2} \mathrm{~S}$ Harding. 'MRC CSO Social and Public Health Sciences Unit, University of Glasgow, Glasgow, UK; ${ }^{2}$ Diabetes and Nutritional Sciences Division, King's College London, London, UK

\subsection{6/jech-2017-SSMAbstracts. 196}

Background There is evidence from existing literature of ethnic variations and clustering in adolescent health risk behaviours. However, it is not known whether there are ethnic variations in health risk behaviour clustering. We aimed to investigate ethnic variations in the clustering of health risk behaviours using the Determinants of young Adults Social wellbeing and Health study (DASH).

Methods Latent class analysis and multinomial logistic regression were used to investigate adolescent health risk behaviour clustering and ethnic variations in clustering in the MRC DASH study. Data were collected from 51 schools across 8 London boroughs. In 2005-06, 4785 pupils were followed up at 14-16 years old. Age, gender, ethnicity, substance use (SU) behaviours (current tobacco and alcohol, and lifetime illicit drug use), daily fruit and vegetables (FV), weekly physical activity (PA), and body mass index (BMI) were recorded.

Results A model with four latent classes was selected (entropy: 0.757). Classes could be characterised as high-SU/high-PA $(\mathrm{n}=232), \quad$ high-SU/low-PA $\quad(\mathrm{n}=811), \quad$ low-SU/PA/high-FV $(\mathrm{n}=1471)$, and low-SU/low-PA/FV $(\mathrm{n}=2260)$.

Using the low-SU/PA/high-FV class as reference males were more likely to be in the high-SU/high-PA class (OR: 1.89; 95\% CI: 1.39-2.57), and less likely to be in the high-SU/lowPA class (OR: 0.56; 95\% CI: 0.47-0.67) than females.

Compared to White UK, Black Caribbean adolescents were less likely to be in the high-SU/high-PA (OR: 0.48; 95\% CI: 0.30-0.76), or high-SU/low-PA (OR: 0.34; 95\% CI: 0.26-0.45) classes. Black Africans were less likely to be in the high-SU/ high-PA (OR: 0.18; 95\% CI: 0.10-0.31), high-SU/low-PA (OR: 0.11 ; 95\% CI: 0.08-0.15), or low-SU/low-PA/FV (OR: 0.55; 95\% CI: 0.44-0.69) classes. Indians were less likely to be in the high-SU/high-PA (OR: 0.36; 95\% CI: 0.19-0.71), or highSU/low-PA (OR: 0.17; 95\% CI: 0.11-0.26) classes. Pakistanis/ Bangladeshis were less likely to be in the high-SU/high-PA (OR: 0.40; 95\% CI: 0.23-0.68), high-SU/low-PA (OR: 0.08; 95\% CI: $0.05-0.13$ ), or low-SU/low-PA/FV (OR: 0.65 ; 95\% CI: 0.50-0.85) classes.

Conclusion Latent class analysis is a valuable method for investigating ethnic variations in adolescent lifestyles. Ethnic minority adolescents tend to be in classes which are characterised by less unhealthy behaviour; patterns also vary between ethnic minority groups. Research needs to investigate risk and protective factors that may explain these ethnic variations to 
identify likely intervention targets and to inform public health policy.

\section{P96 ETHNIC DENSITY EFFECTS FOR ADULT MENTAL HEALTH: SYSTEMATIC REVIEW AND META-ANALYSIS OF INTERNATIONAL STUDIES}

${ }^{1} \mathrm{~L}$ Becares*, ${ }^{2} \mathrm{M}$ Dewey, ${ }^{2} \mathrm{~J}$ Das-Munshi. 'Social Statistics, University of Manchester, Manchester, UK; ${ }^{2}$ Department of Health Services and Population Research, King's College London, Institute of Psychiatry, Psychology and Neuroscience, London, UK

\subsection{6/jech-2017-SSMAbstracts. 197}

Background Despite increased ethnic diversity in more economically developed countries it is unclear whether residential concentration of ethnic minority people (ethnic density) is detrimental or protective for mental health. This is the first systematic review and meta-analysis covering the international literature to assess ethnic density associations with mental health outcomes.

Methods We systematically searched Medline, PsychInfo, Sociological Abstracts, Web of Science from inception to March 31 st, 2016, for observational studies using search terms for the following study outcomes: Depression, anxiety and the common mental disorders, suicide, suicidality, psychotic experiences and psychosis. We obtained additional data from study authors. We conducted random effects meta-analysis taking into account clustering of estimates within datasets. Metaregression assessed heterogeneity in studies due to ethnicity, country, generation and area-level deprivation. This review is registered with PROSPERO CRD42014012992.

Results Out of 2288 studies identified 41 met inclusion criteria. 12 studies provided data for meta-analysis and metaregression. In the meta-analyses, we found a large reduction in relative odds of psychotic experiences (OR: 0.82 (95\% CI: 0.76-0.89) (nine estimates)) and suicidal ideation (OR: 0.88 (95\% CI: 0.79-0.98) (ten estimates)) for each 10 percentagepoint increase in own ethnic density. For common mental disorders, depression and anxiety, associations were indicative of protective effects of own ethnic density however results were not statistically significant.

Conclusion The findings support consistent protective ethnic density associations across countries and racial/ethnic minority populations as well as mental health outcomes. This is indicative of the importance of the social environment in patterning detrimental mental health outcomes in marginalised and excluded population groups.

\section{P97 THE 'PROBLEM' OF ROMA HEALTH AND WELLBEING: A CRITICAL ANALYSIS OF EUROPEAN POLICY PERSPECTIVES}

LC Orton*, S Sheard. Public Health and Policy, University of Liverpool, Liverpool, UK

10.1136/jech-2017-SSMAbstracts. 198

Background Roma are the largest minority ethnic group in Europe. They have faced ongoing discrimination and persecution, with reports of large and widening social and health disparities between Roma and non-Roma. Most analyses of the policies that aim to improve the situation for Europe's Roma compare the pros and cons of proposed solutions. They do not reveal the assumptions that shape how the "problem" of
Roma health and wellbeing is understood in policy proposals and how this influences the solutions that are - or are notput forward. The aim of this study is to explore the conditions that have allowed certain representations of the "problem" of Roma health-and-wellbeing to acquire dominance/ suppression during the 1980-2015 period of economic transition and successive eastward EU expansion.

Methods Five oral history interviews were conducted with European policy specialists to provide accounts of the development of European policy relevant to Roma health and wellbeing and to map key documents produced by EU decisionmaking institutions (the Parliament, Council and Commission). Further documents were located through catalogue searches. All retrieved items were mapped/categorised before five policies relating to pivotal shifts in the way Roma health and wellbeing has been understood as a policy problem were selected. Analyses drew on Bacchi's "What's the problem represented to be?" post-structural analysis framework to explore: 1) underlying frameworks of thought that shape problem representations; 2) the conditions that allowed shifts in problem representations to occur; and 3) how these problem representations shaped the different solutions that have been proposed.

Results Since the 1980's policies produced by the main EU institutions have situated the concern for Roma health and wellbeing within a wider drive for economic growth. A perceived low productivity among Europe's Roma populations is attributed to their low educational attainment and subsequently reduced employment opportunities. Inadequate housing/living conditions and low uptake of health care services are seen to compound the "problem" leaving Roma prone to poor health that further limits their productivity. The proposed solution is to remove the barriers that are perceived to prevent Roma from accessing mainstream health, education, employment and housing.

Conclusion With a perceived increase in the size and mobility of its Roma populations, recent EU policy has framed "Roma health" as a barrier to economic development. Policy solutions frame improvements in Roma health as a means to improve overall productivity of the region, rather than as an end in itself.

\section{P98 ASSESSING CHANGE IN PHYSICAL ACTIVITY LEVELS IN ADOLESCENT ETHNIC GROUPS}

P Bhatnagar*, N Townsend. Nuffield Department of Population Health, University of Oxford, Oxford, UK

\subsection{6/jech-2017-SSMAbstracts. 199}

Background Previous research has established that physical activity declines during childhood, but we have inadequate information on how these changes differ by ethnic group. Determining if inequalities exist between ethnic groups is vital for understanding whether different intervention strategies are necessary. We aimed to determine how physical activity has changed over time within different UK ethnic groups.

Methods We used Understanding Society, a nationally representative UK-based longitudinal survey to track changes in physical activity over time in different ethnic groups. For children aged 10 to 15 , we explored the frequency of participating in sports and mode of travel to school. These factors were measured in four of the available six waves (1, 24 and 6), 